\title{
A Narrative Review of the Relationship between Victimization, Depression and Suicide Ideation among Lesbian, Gay and Bisexual Individuals
}

\author{
MagtumAnak Aen ${ }^{1}$, Norhayatibinti Ibrahim ${ }^{2}$, NormahbintiChe $\operatorname{Din}^{3}$
}

\section{ABSTRACT}

Objective: To study the relationship between victimization, depression, and suicide ideation among lesbian, gay, and bisexual (LGB) individuals. Methods: From a narrative overview of the literature, synthesizing the findings of the relationship between victimization, depression, and suicide ideation among LGB individuals, from a search of relevant database, 3,685 journal articles were retrieved - of which 16 met the study's inclusion criteria. Results and Discussion: Victimization represents a highly stressful experience for the individual involved. Individuals perceived as sexual minorities (i.e., gay, lesbian, bisexual, or transgendered) are specific targets of victimization. Compared to their heterosexual counterparts, sexual minority individuals are more likely to report sexual minority-specific victimization. Sexual minorities are more vulnerable to poor mental health outcomes (e.g., the symptoms of depression) than sexual majorities. This is related to LGBT victimization. Sexual minorities are more likely to report having suicide ideation and attempt than heterosexual individuals. This is associated with LGBT victimization. There is a significant relationship between symptoms of depression and the lifetime history of suicide ideation and attempt among LGBT individuals.

Keywords: Sexual Minority; Victimization; Depression; Suicide; Self-Harm; Risk Behavior

There is a popular consensus among researchers that victimization represents a highly stressful experience for its victim (O’Brennan et al., 2009; Rigby, 2003). In examining the specific targets of victimization, several studies have suggested that individuals perceived as sexual minorities (i.e., gay, lesbian, bisexual, and transgendered) are particularly vulnerable. This is especially true among sexual minority youths (Berlan et al., 2010; Williams et al., 2003). For instance,

\footnotetext{
${ }^{1}$ Master of Clinical Psychology student, Health Psychology Unit, National University of Malaysia. Malaysia, Kuala Lumpur

${ }^{2}$ Senior Lecturer, Health Psychology Unit, National University of Malaysia. Malaysia, Kuala Lumpur

${ }^{3}$ Associate Professor, Health Psychology Unit, National University of Malaysia. Malaysia, Kuala Lumpur

*Responding Author

Received: January 18, 2017; Revision Received: March 18, 2017; Accepted: March 23, 2017

(c) 2017 M Aen, Ibrahim N, Din N; licensee IJIP. This is an Open Access Research distributed under the terms of the Creative Commons Attribution License (www.creativecommons.org/licenses/by/2.0), which permits unrestricted use, distribution, and reproduction in any Medium, provided the original work is properly cited.
} 


\section{A Narrative Review of the Relationship between Victimization, Depression and Suicide Ideation among Lesbian, Gay and Bisexual Individuals}

D’Augelli et al. (2002), in investigating the incidence and mental health impact of sexual orientation victimization of lesbian, gay, and bisexual youths in high school, indicated that LGB youths report more frequent experiences of victimization, e.g., verbal, physical, and homophobic bullying, than their heterosexual peers. According to Harris Interactive, Gay, Lesbian \& Straight Education Network (2005), a survey of school safety among a national sample of students found that LGB students were three times more likely to feel unsafe at school than their heterosexual peers, and $90 \%$ of LGB students reported having been verbally or physically harassed.

Victimization has been linked to compromised emotional health and suicidal ideation (Eisenberg \& Aalsma, 2005). The combination of LGB identity and victimization predicts high levels of mental health problems and risk behaviors (Meyer, 1995). The Minority Stress Model is the most widely adopted theoretical framework in explaining this phenomenon, in which it posits that hegemonic structures upholding heterosexism and homophobia create stressors (e.g., discrimination, stigma, victimization), which may result in mental health issues and risk behaviors among those with minority status (Meyer, 1995). For example, Wilkinson and Person (2009) indicated that youths who attend schools with cultures that are likely to stigmatize LGB youths are more susceptible to mental health problems. Shields et al. (2012), in investigating the impact of victimization on risk of suicide among LGB high school students in San Francisco, stated that LGB individuals who are being victimized have higher odds of attempting suicide or planning to complete suicide. Espelage et al. (2008), in examining homophobic teasing, psychological outcomes, and sexual orientation among high school students, argued that LGB individuals who experience homophobic teasing - including those who question their sexual orientation - are more likely to have depression and suicidal feelings than heterosexual individuals.

It has been suggested that, independent of other suicide risk factors, victimization is a significant risk factor for suicide ideation among LGB individuals (Brunstein et al., 2010; Bontempo et al., 2002). Meanwhile, Silenzio et al. (2007) proposed that suicide ideation among sexual minorities is associated with depression. The purpose of this narrative review is to examine current existing literature and studies in discussing the relationship between victimization, depression, and suicide ideation among lesbian, gay, and bisexual (LGB) individuals.

\section{METHODS}

A comprehensive search of three selected computer databases - EBSCOHOST, Springer, and ScienceDirect - using the search criteria \{LGBT OR "sexual minority" OR homosexual* OR gay OR lesbian OR bisexual AND bully* OR victim* AND depress* OR "mental health" AND suicide* OR "self-harm" OR "risk behavior"\} for the period 2000 to 2015, was conducted. Journal articles were included for the current narrative review, if they met the following criteria: (1) available in full text, (2) the association of sexual minorities or LGB individuals with any of 


\section{A Narrative Review of the Relationship between Victimization, Depression and Suicide Ideation among Lesbian, Gay and Bisexual Individuals}

these factors: victimization, bullying, mental health problems, depression, suicide ideation, and/or risk behaviors, and (3) a sample consisted of adolescents, youths, young adults, and/or adults. For EBSCOHOST, of the 79 studies identified, only 12 met the inclusion criteria. For Springer, the search yielded 2,793 results of which only two were selected. For ScienceDirect, the search yielded 813 results of which only two were selected. Overall, 16 journal articles that met the inclusion criteria were chosen for the current narrative review.

\section{RESULTS}

\begin{tabular}{|c|c|c|c|c|}
\hline \multirow[b]{2}{*}{2012} & Sample & Test & Design & Results \\
\hline & $\begin{array}{l}\text { United States } \\
\text { of America; } \\
\text { 237 LGBT } \\
\text { participants; } \\
\text { 16-20 years } \\
\text { old; } 47.7 \% \\
\text { males, 52.3\%; } \\
61.6 \% \\
\text { gay/lesbian, } \\
28.7 \% \\
\text { bisexual, and } \\
9.7 \% \text { other } \\
\text { (i.e., } \\
\text { questioning, } \\
\text { queer, } \\
\text { unsure). }\end{array}$ & $\begin{array}{l}\text { Diagnostic Interview } \\
\text { Schedule for Children } \\
\text { (DISC) computerized } \\
\text { version } 4.0 \\
\text { 6-item Brief } \\
\text { Hopelessness Scale } \\
\text { (adapted from } \\
\text { Hopelessness Scale } \\
\text { for Children) } \\
\text { Barratt Impulsiveness } \\
\text { Scale (BIS-11) } \\
\text { The Multidimensional } \\
\text { Scale of Perceived } \\
\text { Social Support } \\
\text { (MSPSS) } \\
\text { 5-item Boyhood } \\
\text { Gender Conformity } \\
\text { Scale in males and a } \\
\text { validated 4-item } \\
\text { adaptation for girls. } \\
\text { An item from the } \\
\text { Sexual Risk Behavior } \\
\text { Assessment Schedule } \\
\text { for Homosexual } \\
\text { Youths. } \\
\text { A 10-item measure } \\
\text { assessed the } \\
\text { frequency of lifetime } \\
\text { experiences of } \\
\text { victimization } \\
\text { "because you are, or }\end{array}$ & $\begin{array}{l}\text { Questionnaires } \\
\text { (measuring } \\
\text { general risk } \\
\text { factors for } \\
\text { suicide } \\
\text { attempts: } \\
\text { hopelessness, } \\
\text { impulsiveness, } \\
\text { and perceived } \\
\text { social support; } \\
\text { LGBT specific } \\
\text { suicide risk } \\
\text { factors: gender } \\
\text { nonconformity, } \\
\text { age of first } \\
\text { same-sex } \\
\text { attraction, and } \\
\text { LGBT } \\
\text { victimization) }\end{array}$ & $\begin{array}{l}\text { Out of } 9 \text { variables } \\
\text { examined, } 7 \text { were } \\
\text { related to lifetime } \\
\text { history of attempted } \\
\text { suicide: } \\
\text { hopelessness, } \\
\text { depression } \\
\text { symptoms, conduct } \\
\text { disorder symptoms, } \\
\text { impulsivity, } \\
\text { victimization, age of } \\
\text { first same-sex } \\
\text { attraction, and low } \\
\text { family support. } \\
\text { Depressive } \\
\text { symptoms and } \\
\text { hopelessness } \\
\text { mediated the } \\
\text { relation between } \\
\text { multiple risk and } \\
\text { resilience factors } \\
\text { and suicide } \\
\text { attempts. }\end{array}$ \\
\hline
\end{tabular}

(C) The International Journal of Indian Psychology, ISSN 2348-5396 (e)| ISSN: 2349-3429 (p) | 96 


\section{A Narrative Review of the Relationship between Victimization, Depression and Suicide Ideation among Lesbian, Gay and Bisexual Individuals}

\begin{tabular}{|c|c|c|c|c|}
\hline Reference & Sample & Test & Design & Results \\
\hline & & $\begin{array}{l}\text { were thought to be, } \\
\text { gay, lesbian, bisexual, } \\
\text { or transgender'’. }\end{array}$ & & $\begin{array}{l}\text { greater odds of } \\
\text { making another } \\
\text { attempt in the 1- } \\
\text { year follow-up } \\
\text { period than were } \\
\text { those who had made } \\
\text { no previous attempt. }\end{array}$ \\
\hline $\begin{array}{l}\text { Russell \& Joyner, } \\
2001\end{array}$ & $\begin{array}{l}\text { United States } \\
\text { of America; } \\
6254 \\
\text { adolescent } \\
\text { girls and } 5686 \\
\text { adolescents; } \\
\text { same-sex } \\
\text { relationships: } \\
\text { l.1\% of boys } \\
\text { and } 2.0 \% \text { of } \\
\text { girls, same- } \\
\text { sex romantic } \\
\text { attractions: } \\
7.3 \% \text { of boys } \\
\text { and 5\% of } \\
\text { girls, both } \\
\text { same-sex } \\
\text { romantic } \\
\text { attraction and } \\
\text { relationship: } \\
0.5 \% \text { of boys } \\
\text { and girls. }\end{array}$ & $\begin{array}{l}\text { Two dichotomous } \\
\text { reports of suicidal } \\
\text { thoughts and } \\
\text { behaviors. } \\
\text { Hopelessness was } \\
\text { measured with a } \\
\text { single item: "You felt } \\
\text { hopeful about the } \\
\text { future.” } \\
\text { Center for } \\
\text { Epidemiologic } \\
\text { Studies - Depression } \\
\text { inventory (CES-D) } \\
\text { Alcohol abuse was } \\
\text { measured with the } \\
\text { sum of } 9 \text { items that } \\
\text { indicate problems } \\
\text { associated with } \\
\text { alcohol use during the } \\
\text { past } 12 \text { months. } \\
\text { Two questions } \\
\text { pertained to recent } \\
\text { experiences with } \\
\text { suicide. } \\
\text { Victimization was } \\
\text { measured with } \\
\text { affirmative responses } \\
\text { to any of the } \\
\text { following } 4 \text { items: } \\
\text { "Someone pulled a } \\
\text { knife or gun on you,” } \\
\text { "You were jumped,”, } \\
\text { "Someone shot you,” } \\
\text { and "Someone cut or } \\
\text { stabbed you.” }\end{array}$ & $\begin{array}{l}\text { Audio } \\
\text { computer-aided } \\
\text { self-interview } \\
\text { Survey }\end{array}$ & $\begin{array}{l}\text { There is a strong } \\
\text { link between } \\
\text { adolescent sexual } \\
\text { orientation and } \\
\text { suicidal thoughts } \\
\text { and behaviors. } \\
\text { The strong effect of } \\
\text { sexual orientation } \\
\text { on suicidal thoughts } \\
\text { is mediated by } \\
\text { critical youth } \\
\text { suicide risk factors, } \\
\text { including } \\
\text { depression, } \\
\text { hopelessness, } \\
\text { alcohol abuse, } \\
\text { recent suicide } \\
\text { attempts by a peer } \\
\text { or a family member, } \\
\text { and experiences of } \\
\text { victimization. }\end{array}$ \\
\hline
\end{tabular}


A Narrative Review of the Relationship between Victimization, Depression and Suicide Ideation among Lesbian, Gay and Bisexual Individuals

\begin{tabular}{|c|c|c|c|c|}
\hline Reference & Sample & Test & Design & Results \\
\hline Patrick et al., 2013 & $\begin{array}{l}\text { United States } \\
\text { of America; } \\
27,752 \\
\text { adolescents in } \\
\text { public school } \\
\text { grades } 8,10 \text {, } \\
\text { and } 12 \text {. }\end{array}$ & $\begin{array}{l}\text { Bullying - A binary } \\
\text { measure derived from } \\
\text { the survey question: } \\
\text { "In the past } 30 \text { days, } \\
\text { how often were you } \\
\text { bullied, harassed, or } \\
\text { intimidated at school } \\
\text { or on your way to or } \\
\text { from school because } \\
\text { someone thought you } \\
\text { were gay, lesbian or } \\
\text { bisexual (whether you } \\
\text { are or are not)?” } \\
\text { Youth QOL } \\
\text { Instrument - Healthy } \\
\text { Youth Survey } \\
\text { Version (YQOL - } \\
\text { HYS) } \\
\text { Depression and } \\
\text { suicide ideation - A } \\
\text { binary measure of } \\
\text { depressed mood was } \\
\text { derived from the } \\
\text { question “During the } \\
\text { past } 12 \text { months, did } \\
\text { you ever feel so sad } \\
\text { or hopeless almost } \\
\text { every day for } 2 \text { weeks } \\
\text { or more in a row that } \\
\text { you stopped doing } \\
\text { some usual } \\
\text { activities?” Students } \\
\text { who answered “yes” } \\
\text { were asked about } \\
\text { suicide ideation: } \\
\text { "During the past } 12 \\
\text { months, did you ever } \\
\text { seriously consider } \\
\text { attempting suicide?” }\end{array}$ & Survey & $\begin{array}{l}\text { Among male } \\
\text { students, 14\%, 11\%, } \\
\text { and 9\% reported } \\
\text { being bullied } \\
\text { because of } \\
\text { perceived sexual } \\
\text { orientation in 8th, } \\
\text { 10th, and 12th } \\
\text { grades, respectively; } \\
\text { and among female } \\
\text { students, 11\%, 10\%, } \\
\text { and 6\%. } \\
\text { In all gender and } \\
\text { grade strata, being } \\
\text { bullied because of } \\
\text { PSO was associated } \\
\text { with lower quality } \\
\text { of life scores and } \\
\text { increased the odds } \\
\text { of depressed mood } \\
\text { or consideration of } \\
\text { suicide. Moreover, } \\
\text { the magnitudes of } \\
\text { these associations } \\
\text { were greater than } \\
\text { for being bullied for } \\
\text { other reasons. }\end{array}$ \\
\hline Walls et al., 2012 & $\begin{array}{l}\text { United States } \\
\text { of America; } \\
\text { sexual } \\
\text { minority } \\
\text { youths and }\end{array}$ & $\begin{array}{l}\text { Two questions } \\
\text { regarding } \\
\text { victimization: (1) } \\
\text { Physical harassment } \\
\text { and attack by a family }\end{array}$ & Survey & $\begin{array}{l}\text { Prevalence of } \\
\text { cutting was higher } \\
\text { than that found in } \\
\text { community-based } \\
\text { samples of similar }\end{array}$ \\
\hline
\end{tabular}


A Narrative Review of the Relationship between Victimization, Depression and Suicide Ideation among Lesbian, Gay and Bisexual Individuals

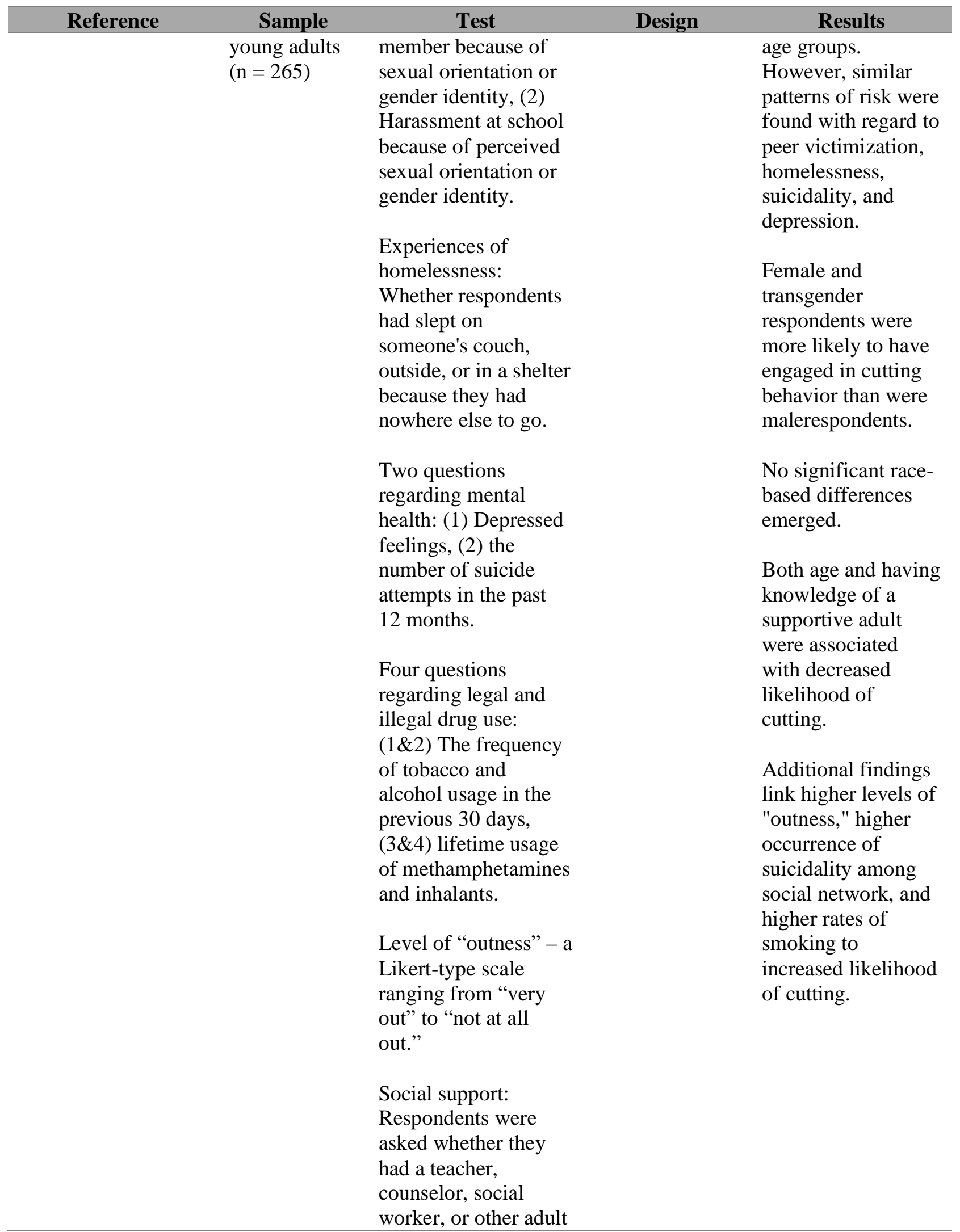


A Narrative Review of the Relationship between Victimization, Depression and Suicide Ideation among Lesbian, Gay and Bisexual Individuals

\begin{tabular}{|c|c|c|c|c|}
\hline Reference & Sample & Test & Design & Results \\
\hline & & $\begin{array}{l}\text { in their school or } \\
\text { college who they felt } \\
\text { safe talking to about } \\
\text { their sexual } \\
\text { orientation or gender } \\
\text { identity. } \\
\text { A question about the } \\
\text { prevalence of suicide } \\
\text { among the } \\
\text { participant's network } \\
\text { of friends. } \\
\text { Two questions } \\
\text { regarding cutting } \\
\text { behavior: (1) } \\
\text { Frequency, (2) } \\
\text { Activities helped the } \\
\text { respondent refrain } \\
\text { from engaging in } \\
\text { cutting behavior. }\end{array}$ & & \\
\hline $\begin{array}{l}\text { Blosnich\&Bossarte, } \\
2012\end{array}$ & $\begin{array}{l}\text { United States } \\
\text { of America; a } \\
\text { national } \\
\text { sample of } \\
\text { college- } \\
\text { attending 18- } \\
\text { to 24-year- } \\
\text { olds, n = } \\
\text { 8,576; male = } \\
\text { 3,150, female } \\
=5,426 \text {; } \\
\text { gay/lesbian = } \\
21 \% \text {, bisexual } \\
=29.2 \% \text {, } \\
\text { heterosexual = } \\
49.8 \% \text {. }\end{array}$ & $\begin{array}{l}\text { Violence-related } \\
\text { stressors: Reports of } \\
\text { experiencing physical } \\
\text { assault, sexual assault } \\
\text { or intimate partner } \\
\text { violence in the last } 12 \\
\text { months. } \\
\text { Familial strain: A } \\
\text { question that asked } \\
\text { respondents if, in the } \\
\text { past } 12 \text { months, they } \\
\text { had family problems } \\
\text { that were either } \\
\text { traumatic or very } \\
\text { difficult to handle. } \\
\text { One item asked } \\
\text { respondents whether } \\
\text { they had experienced } \\
\text { discrimination in the } \\
\text { last } 12 \text { months and } \\
\text { whether it affected } \\
\text { their academic } \\
\text { performance and to } \\
\text { what degree it }\end{array}$ & $\begin{array}{l}\text { Survey } \\
\text { (paper-and- } \\
\text { pencil or Web } \\
\text { based format or } \\
\text { both) }\end{array}$ & $\begin{array}{l}\text { Sexual minorities } \\
\text { reported more } \\
\text { socially based } \\
\text { stressors than } \\
\text { heterosexuals. } \\
\text { Significantly higher } \\
\text { prevalence of self- } \\
\text { injurious behavior, } \\
\text { suicide ideation, and } \\
\text { suicide attempt } \\
\text { among sexual } \\
\text { minorities relative } \\
\text { to their heterosexual } \\
\text { peers. } \\
\text { Bisexuals exhibited } \\
\text { greatest prevalence } \\
\text { of self-injurious and } \\
\text { suicidal behaviors. } \\
\text { In adjusted models, } \\
\text { intimate partner } \\
\text { violence was most } \\
\text { consistently } \\
\text { associated with self- }\end{array}$ \\
\hline
\end{tabular}

(c) The International Journal of Indian Psychology, ISSN 2348-5396 (e)| ISSN: 2349-3429 (p) | 100 
A Narrative Review of the Relationship between Victimization, Depression and Suicide Ideation among Lesbian, Gay and Bisexual Individuals

\begin{tabular}{|c|c|c|c|c|}
\hline Reference & Sample & Test & Design & Results \\
\hline & & $\begin{array}{l}\text { affected their } \\
\text { schooling. } \\
\text { Mental health: Self- } \\
\text { injurious behavior, } \\
\text { suicide ideation, and } \\
\text { suicide attempt in the } \\
\text { last } 12 \text { months. }\end{array}$ & & injurious behaviors. \\
\hline $\begin{array}{l}\text { LeVasseur et al., } \\
2013\end{array}$ & $\begin{array}{l}\text { United States } \\
\text { of America; } \\
11,488 \\
\text { respondents; } \\
\text { male = 47.8\%, } \\
\text { female = } \\
52.2 \% \text {; sexual } \\
\text { minority = } \\
7.9 \% \text {. }\end{array}$ & $\begin{array}{l}\text { Suicide attempt: } \\
\text { "During the past } 12 \\
\text { months, how many } \\
\text { times did you actually } \\
\text { attempt suicide?” } \\
\text { School bullying: } \\
\text { "During the past } 12 \\
\text { months, have you } \\
\text { ever been } \\
\text { bullied on school } \\
\text { property?” }\end{array}$ & Survey & $\begin{array}{l}\text { Compared with } \\
\text { non-sexual minority } \\
\text { youths, sexual } \\
\text { minority youths had } \\
4.39 \text { and } 1.96 \text { times } \\
\text { higher odds, } \\
\text { respectively, of } \\
\text { attempting suicide } \\
\text { and reporting } \\
\text { bullying. } \\
\text { Those who reported } \\
\text { bullying, who } \\
\text { identified as sexual } \\
\text { minorities, who } \\
\text { identified as } \\
\text { Hispanic, and who } \\
\text { were female were } \\
\text { more likely to report } \\
\text { suicide attempt. } \\
\text { These identities } \\
\text { appear to interact } \\
\text { with one another to } \\
\text { modify the } \\
\text { relationship } \\
\text { between reported } \\
\text { bullying and } \\
\text { reported suicide } \\
\text { attempt. }\end{array}$ \\
\hline Russell et al., 2011 & $\begin{array}{l}\text { United States } \\
\text { of America; } \\
245 \\
\text { California- } \\
\text { based LGBT } \\
\text { young adults } \\
\text { between the } \\
\text { ages of } 21 \text { and }\end{array}$ & $\begin{array}{l}\text { A } 10 \text {-item } \\
\text { retrospective scale } \\
\text { assessed school } \\
\text { victimization due to } \\
\text { actual or perceived } \\
\text { LGBT status between } \\
\text { the ages of } 13 \text { and } 19 \\
\text { years (adapted from }\end{array}$ & Survey & $\begin{array}{l}\text { Lesbian, gay, } \\
\text { bisexual, and } \\
\text { transgender-related } \\
\text { school victimization } \\
\text { is strongly linked to } \\
\text { young adult mental } \\
\text { health and risk for } \\
\text { STDs and HIV; }\end{array}$ \\
\hline
\end{tabular}

(c) The International Journal of Indian Psychology, ISSN 2348-5396 (e)| ISSN: 2349-3429 (p) | 101 
A Narrative Review of the Relationship between Victimization, Depression and Suicide Ideation among Lesbian, Gay and Bisexual Individuals

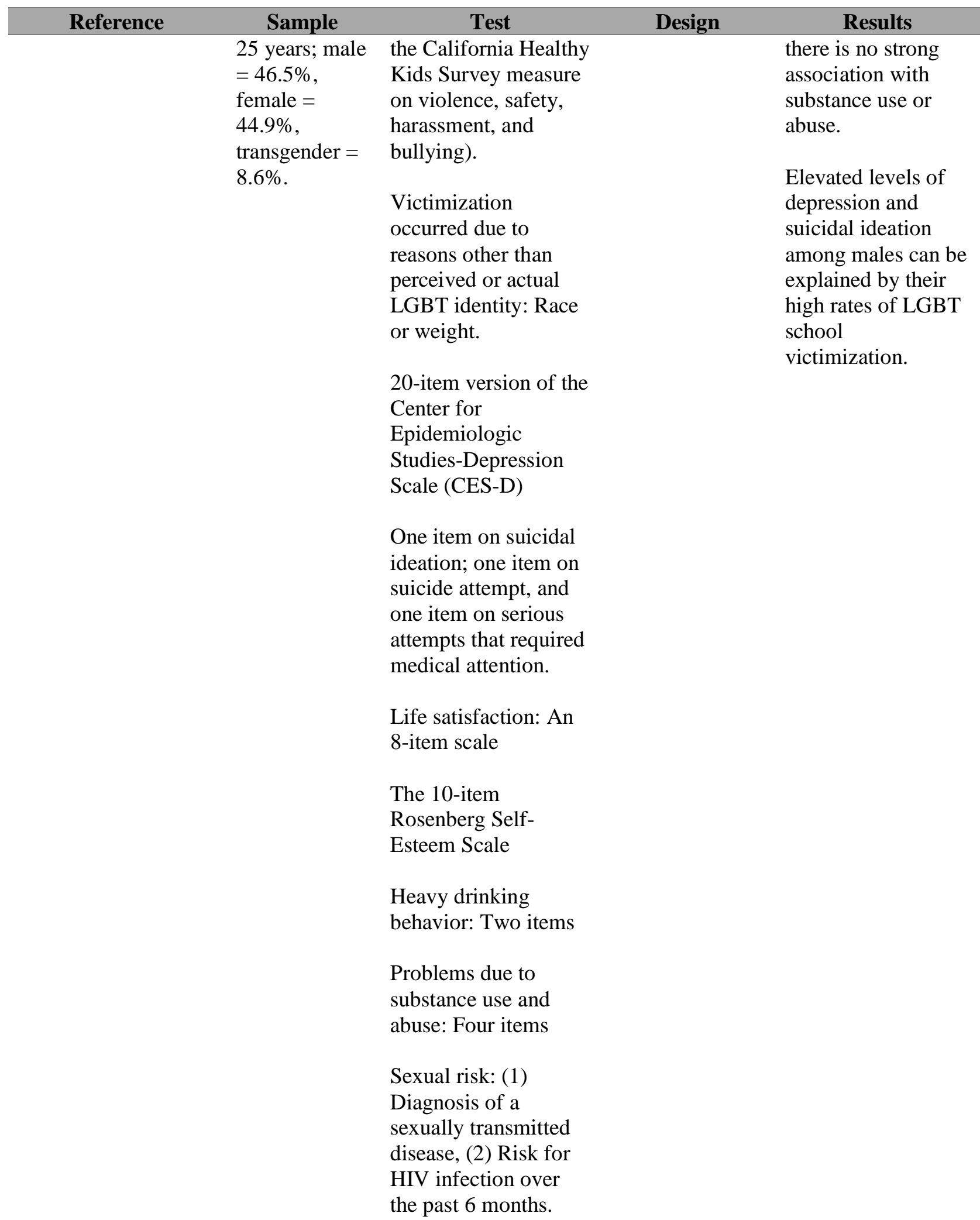




\section{A Narrative Review of the Relationship between Victimization, Depression and Suicide Ideation among Lesbian, Gay and Bisexual Individuals}

\begin{tabular}{|c|c|c|c|c|}
\hline Reference & Sample & Test & Design & Results \\
\hline $\begin{array}{l}\text { Hightow-Weidman et } \\
\text { al., } 2011\end{array}$ & $\begin{array}{l}\text { United States } \\
\text { of America; } \\
351 \\
\text { racial/ethnic } \\
\text { minority } \\
\text { young men } \\
\text { who have sex } \\
\text { with men } \\
\text { (YMSM); } \\
\text { mean age = } \\
20.4 \text {; gay = } \\
65.5 \% \text {, } \\
\text { bisexual = } \\
20.5 \% \text {; } \\
\text { African- } \\
\text { American = } \\
67.5 \% \text {, Latino } \\
=20.2 \% \text {, } \\
\text { multiracial = } \\
12.3 \% \text {. }\end{array}$ & $\begin{array}{l}\text { Racial bullying: Two } \\
\text { items } \\
\text { Sexuality-related } \\
\text { bullying: Three items } \\
\text { The Center for } \\
\text { Epidemiologic } \\
\text { Studies Depression } \\
\text { Scale (CES-D) } \\
\text { Two other indicators } \\
\text { to measure } \\
\text { emotional distress: (1) } \\
\text { Suicidal ideation, (2) } \\
\text { Prior acts of self- } \\
\text { harm. } \\
\text { Support from family: } \\
\text { Two items } \\
\text { Support from close } \\
\text { friends: One item } \\
\text { Parental abuse: One } \\
\text { item }\end{array}$ & Survey & $\begin{array}{l}\text { Overall } 36 \% \text { and } \\
\text { 85\% of participants } \\
\text { experienced racial } \\
\text { and sexuality- } \\
\text { related bullying, } \\
\text { respectively. } \\
\text { There was a } \\
\text { significant } \\
\text { association between } \\
\text { experiencing a high } \\
\text { level of sexuality- } \\
\text { related bullying and } \\
\text { depressive } \\
\text { symptomatology (p } \\
=0.03 \text { ), having } \\
\text { attempted suicide (p } \\
=0.03 \text { ), and } \\
\text { reporting parental } \\
\text { abuse (p = 0.05). } \\
\text { No association } \\
\text { between racial } \\
\text { bullying and suicide } \\
\text { attempts was found. } \\
\text { In a multivariable } \\
\text { logistic regression } \\
\text { model, experiencing } \\
\text { any racial bullying } \\
\text { and high sexuality- } \\
\text { related bullying } \\
\text { were significant } \\
\text { predictors of having } \\
\text { a CES-D (The } \\
\text { Center for } \\
\text { Epidemiologic } \\
\text { Studies Depression } \\
\text { Scale) score } \geq 16 ; \\
\text { adjusted odds ratio } \\
\text { (OR) } 1.83 \text { and } 2.29 \text {, } \\
\text { respectively. }\end{array}$ \\
\hline Burton et al., 2013 & $\begin{array}{l}\text { United States } \\
\text { of America; } \\
192 \\
\text { adolescents } \\
\text { ranging in age }\end{array}$ & $\begin{array}{l}\text { Victimization due to } \\
\text { actual or perceived } \\
\text { sexual minority status } \\
\text { was measured at } \\
\text { waves } 1 \text { and } 2 \text { by four }\end{array}$ & $\begin{array}{l}\text { Survey } \\
\text { Longitudinal } \\
\text { study }\end{array}$ & $\begin{array}{l}\text { Compared to } \\
\text { heterosexual youth, } \\
\text { sexual minority } \\
\text { youth reported } \\
\text { higher levels of }\end{array}$ \\
\hline
\end{tabular}

(c) The International Journal of Indian Psychology, ISSN 2348-5396 (e) | ISSN: 2349-3429 (p) | 103 


\section{A Narrative Review of the Relationship between Victimization, Depression and Suicide Ideation among Lesbian, Gay and Bisexual Individuals}

\begin{tabular}{|c|c|c|c|c|}
\hline Reference & Sample & Test & Design & Results \\
\hline & $\begin{array}{l}\text { from } 14 \text { to 19; } \\
\text { sexual } \\
\text { minority = } \\
29 \% \text { (female } \\
=25 \% \text {, male = } \\
4 \% \text {, White = } \\
6 \% \text {, } \\
\text { racial/ethnic } \\
\text { minority = } \\
23 \% \text { ), } \\
\text { heterosexual = } \\
71 \% \text { (female } \\
=45 \% \text {, male = } \\
50 \% \text {, White = } \\
28 \%, \\
\text { racial/ethnic } \\
\text { minority = } \\
43 \% \text { ). }\end{array}$ & $\begin{array}{l}\text { items that assessed } \\
\text { the frequency during } \\
\text { the past } 6 \text { months of } \\
\text { being teased/bullied, } \\
\text { hit/beaten up, treated } \\
\text { unfairly, or called bad } \\
\text { names because } \\
\text { someone thought the } \\
\text { participant was } \\
\text { gay/lesbian. } \\
\text { Center for } \\
\text { Epidemiologic } \\
\text { Studies Depression } \\
\text { Scale (CES-D) } \\
\text { Lifetime history of } \\
\text { suicidal thoughts and } \\
\text { intent: Wave } 1 \text { "Have } \\
\text { you ever thought } \\
\text { about or attempted to } \\
\text { kill yourself?”, Wave } \\
2 \text { “In the past } 6 \\
\text { months have you ever } \\
\text { thought about or } \\
\text { attempted to kill } \\
\text { yourself?” }\end{array}$ & & $\begin{array}{l}\text { sexual minority- } \\
\text { specific } \\
\text { victimization, } \\
\text { depressive } \\
\text { symptoms, and } \\
\text { suicidality. } \\
\text { Sexual minority- } \\
\text { specific } \\
\text { victimization } \\
\text { significantly } \\
\text { mediated the effect } \\
\text { of sexual minority } \\
\text { status on depressive } \\
\text { symptoms and } \\
\text { suicidality. } \\
\text { The results support } \\
\text { the minority stress } \\
\text { hypothesis that } \\
\text { targeted harassment } \\
\text { and victimization } \\
\text { are partly } \\
\text { responsible for the } \\
\text { higher levels of } \\
\text { depressive } \\
\text { symptoms and } \\
\text { suicidality found in } \\
\text { sexual minority } \\
\text { youth. }\end{array}$ \\
\hline Mueller et al., 2015 & $\begin{array}{l}\text { United States } \\
\text { of America; } \\
75,344 \\
\text { adolescents; } \\
\text { heterosexual = } \\
\text { 93.49\%, gay } \\
\text { or lesbian = } \\
1.76 \% \text {, } \\
\text { bisexual = } \\
4.76 \% \text {; White } \\
=49.40 \% \text {, } \\
\text { Black= } \\
\text { 19.8\%, } \\
\text { Hispanic = } \\
30.80 \%\end{array}$ & $\begin{array}{l}\text { Suicide ideation: } \\
\text { "During the past } 12 \\
\text { months, did you ever } \\
\text { seriously consider } \\
\text { attempting suicide?” } \\
\text { Bullying: (1) "During } \\
\text { the past } 12 \text { months, } \\
\text { have you been bullied } \\
\text { on school property?”, } \\
\text { (2) “During the past } \\
12 \text { months, have you } \\
\text { ever been } \\
\text { electronically bullied } \\
\text { (include being bullied } \\
\text { through e-mail, chat } \\
\text { rooms, instant }\end{array}$ & Survey & $\begin{array}{l}\text { White and Hispanic } \\
\text { gay and bisexual } \\
\text { males, white lesbian } \\
\text { and bisexual } \\
\text { females, and } \\
\text { Hispanic bisexual } \\
\text { females were more } \\
\text { likely to be bullied } \\
\text { than were white } \\
\text { heterosexual } \\
\text { adolescents. } \\
\text { Black lesbian, gay, } \\
\text { and bisexual youths' } \\
\text { vulnerability to } \\
\text { being bullied was } \\
\text { not significantly }\end{array}$ \\
\hline
\end{tabular}

(C) The International Journal of Indian Psychology, ISSN 2348-5396 (e)| ISSN: 2349-3429 (p) | 104 
A Narrative Review of the Relationship between Victimization, Depression and Suicide Ideation among Lesbian, Gay and Bisexual Individuals

\begin{tabular}{|c|c|c|c|c|}
\hline Reference & Sample & Test & Design & Results \\
\hline & & $\begin{array}{l}\text { messaging, Web sites, } \\
\text { or texting)?” }\end{array}$ & & $\begin{array}{l}\text { different from that } \\
\text { of white } \\
\text { heterosexual youths. } \\
\text { Black and Hispanic } \\
\text { heterosexual youths } \\
\text { were less likely to } \\
\text { be bullied than were } \\
\text { white heterosexual } \\
\text { youths. } \\
\text { Despite differences } \\
\text { in the likelihood of } \\
\text { being bullied, } \\
\text { sexual minority } \\
\text { youths were more } \\
\text { likely to report } \\
\text { suicide ideation, } \\
\text { regardless of their } \\
\text { race/ethnicity, their } \\
\text { gender, or whether } \\
\text { they have been } \\
\text { bullied. }\end{array}$ \\
\hline Ferlatte et al., 2015 & $\begin{array}{l}\text { Canada; 8,382 } \\
\text { Canadian } \\
\text { men; gay = } \\
64.5 \% \text {, } \\
\text { bisexual = } \\
32.5 \% \text {, } \\
\text { heterosexual = } \\
2.1 \% \text {, other = } \\
1.0 \% \text {. }\end{array}$ & $\begin{array}{l}\text { Suicide related } \\
\text { ideation and behavior: } \\
\text { (1) “Have you ever } \\
\text { thought about } \\
\text { suicide?”, (2) "Have } \\
\text { you ever attempted } \\
\text { suicide?”. (prior to } \\
\text { the last } 12 \text { months, } \\
\text { within the last } 12 \\
\text { months, or both prior } \\
\text { to and within the last } \\
12 \text { months). } \\
\text { Lifetime experiences } \\
\text { of anti-gay } \\
\text { marginalization and } \\
\text { violence: (1) Verbal } \\
\text { violence and/or hate } \\
\text { talk, (2) Physical } \\
\text { violence, (3) Anti-gay } \\
\text { bullying (i.e. } \\
\text { harassment, cyber- } \\
\text { bullying), (4) Sexual } \\
\text { violence (i.e. }\end{array}$ & Survey & $\begin{array}{l}\text { Suicide ideation and } \\
\text { attempts were } \\
\text { positively } \\
\text { associated with each } \\
\text { individual } \\
\text { marginalization } \\
\text { indicator (verbal } \\
\text { violence, physical } \\
\text { violence, bullying, } \\
\text { sexual violence and } \\
\text { work } \\
\text { discrimination) and } \\
\text { psychosocial health } \\
\text { problems (smoking, } \\
\text { party drugs, } \\
\text { depression, anxiety, } \\
\text { STIs, HIV risk and } \\
\text { HIV). } \\
\text { Furthermore, } \\
\text { prevalence of } \\
\text { suicide ideation and } \\
\text { attempts increased } \\
\text { with each added }\end{array}$ \\
\hline
\end{tabular}

(c) The International Journal of Indian Psychology, ISSN 2348-5396 (e) | ISSN: 2349-3429 (p) | 105 
A Narrative Review of the Relationship between Victimization, Depression and Suicide Ideation among Lesbian, Gay and Bisexual Individuals

\begin{tabular}{|c|c|c|c|c|}
\hline Reference & Sample & Test & Design & Results \\
\hline & & $\begin{array}{l}\text { unwanted sex), and } \\
\text { (5) workplace } \\
\text { discrimination based } \\
\text { on their sexuality. } \\
\text { Psychosocial health } \\
\text { problems: } \\
\text { Experiences in the } \\
\text { last } 12 \text { months: (1) } \\
\text { Frequent } \\
\text { consumption of } \\
\text { tobacco (regular/daily } \\
\text { smokers), (2) Use of } \\
\text { one or multiple of the } \\
\text { following party drugs, } \\
\text { (3) Being on } \\
\text { medication for } \\
\text { depression, (4) Being } \\
\text { on medication for } \\
\text { anxiety, (5) Being } \\
\text { diagnosed with one or } \\
\text { more sexually } \\
\text { transmitted infections } \\
\text { (STIs), (6) One or } \\
\text { more episodes of } \\
\text { condomless anal } \\
\text { intercourse (insertive } \\
\text { or receptive) with a } \\
\text { partner (CAI-US), } \\
\text { whose HIV status was } \\
\text { unknown or } \\
\text { discordant, and (7) } \\
\text { Have ever been } \\
\text { diagnosed with HIV. }\end{array}$ & & $\begin{array}{l}\text { psychosocial health } \\
\text { problem. } \\
\text { Those who reported } \\
3 \text { or more had } 6.90 \\
\text { (5.47-8.70) times } \\
\text { the odds of } \\
\text { experiencing suicide } \\
\text { ideation and } 16.29 \\
\text { (9.82-27.02) times } \\
\text { the odds of a suicide } \\
\text { attempt compared to } \\
\text { those with no } \\
\text { psychosocial health } \\
\text { problems. }\end{array}$ \\
\hline \multirow[t]{2}{*}{ Bergen et al., 2013} & $\begin{array}{l}\text { Netherlands; } \\
274 \text { Dutch } \\
\text { lesbian, gay, } \\
\text { and bisexual } \\
\text { youths. }\end{array}$ & $\begin{array}{l}\text { Lifetime suicidal } \\
\text { ideation and suicide } \\
\text { attempts were each } \\
\text { measured with } 1 \text { item } \\
\text { (1 = no; } 2 \text { = yes). }\end{array}$ & Survey & $\begin{array}{l}\text { Suicidality among } \\
\text { Dutch LGB youths } \\
\text { is significantly } \\
\text { higher than among } \\
\text { heterosexual youths. }\end{array}$ \\
\hline & & $\begin{array}{l}\text { Experiences of } \\
\text { homophobic } \\
\text { victimization in the } \\
\text { preceding } 12 \text { months } \\
\text { by parents, family } \\
\text { members outside the } \\
\text { nuclear family, at }\end{array}$ & & $\begin{array}{l}\text { The impact of } \\
\text { victimization on } \\
\text { suicidality depends } \\
\text { on the context in } \\
\text { which the } \\
\text { victimization takes } \\
\text { place. Negative }\end{array}$ \\
\hline
\end{tabular}

(c) The International Journal of Indian Psychology, ISSN 2348-5396 (e)| ISSN: 2349-3429 (p) | 106 


\section{A Narrative Review of the Relationship between Victimization, Depression and Suicide Ideation among Lesbian, Gay and Bisexual Individuals}

\begin{tabular}{|c|c|c|c|c|}
\hline Reference & Sample & Test & Design & Results \\
\hline & & $\begin{array}{l}\text { school, and by people } \\
\text { in the neighborhood } \\
\text { were measured with } \\
\text { 1-item questions. }\end{array}$ & & $\begin{array}{l}\text { reactions at school } \\
\text { were related to both } \\
\text { suicidal ideation } \\
\text { and suicide } \\
\text { attempts, whereas } \\
\text { negative reactions } \\
\text { by parents were } \\
\text { only related to } \\
\text { suicide attempts. } \\
\text { Although negative } \\
\text { reactions from } \\
\text { parents were related } \\
\text { to suicidality among } \\
\text { LGB youths, it } \\
\text { should be noted that } \\
\text { victimization at } \\
\text { school was the } \\
\text { strongest predictor. }\end{array}$ \\
\hline $\begin{array}{l}\text { Muehlenkamp et al., } \\
2015\end{array}$ & $\begin{array}{l}\text { United States } \\
\text { of America; } \\
137 \text { college } \\
\text { students who } \\
\text { were at least } \\
18 \text { years old, } \\
\text { identified as a } \\
\text { sexual } \\
\text { minority. }\end{array}$ & $\begin{array}{l}\text { Schedule of Sexually } \\
\text { Discriminatory } \\
\text { Events (SSDE; } \\
\text { House, Coppeans, \& } \\
\text { Stepleman: The } \\
\text { Schedule ofSexually } \\
\text { Discriminatory } \\
\text { Events,unpublished) } \\
\text { Expectation of } \\
\text { rejection: A self- } \\
\text { designed series of } \\
\text { questions asking } \\
\text { participants about } \\
\text { their degree of worry } \\
\text { related to expecting } \\
\text { negative interactions. } \\
\text { Interpersonal Needs } \\
\text { Questionnaire } \\
\text { Acquired Capability } \\
\text { Scale }\end{array}$ & Survey & $\begin{array}{l}\text { Sexual minorities } \\
\text { who experience } \\
\text { minority stress may } \\
\text { be at elevated risk } \\
\text { for non-suicidal } \\
\text { self-injury (NSSI), } \\
\text { especially if they } \\
\text { also perceive } \\
\text { themselves to be a } \\
\text { burden on others. } \\
\text { Subsequently, } \\
\text { having engaged in } \\
\text { NSSI appears to } \\
\text { confer increased } \\
\text { risk for suicidal } \\
\text { thoughts and } \\
\text { behaviors both } \\
\text { directly and by } \\
\text { increasing acquired } \\
\text { capability. }\end{array}$ \\
\hline
\end{tabular}

(c) The International Journal of Indian Psychology, ISSN 2348-5396 (e) | ISSN: 2349-3429 (p) | 107 


\section{A Narrative Review of the Relationship between Victimization, Depression and Suicide Ideation among Lesbian, Gay and Bisexual Individuals}

\begin{tabular}{|c|c|c|c|c|}
\hline \multirow{2}{*}{$\begin{array}{c}\text { Reference } \\
\text { King et al., } 2008\end{array}$} & Sample & Test & Design & Results \\
\hline & $\begin{array}{l}\text { United } \\
\text { Kingdom; } \\
\text { data was } \\
\text { extracted on } \\
214,344 \\
\text { heterosexuals } \\
\text { and } 11,971 \\
\text { non- } \\
\text { heterosexual } \\
\text { people. }\end{array}$ & $\begin{array}{l}\text { Databases: Medline, } \\
\text { Embase, PsycInfo, } \\
\text { Cinahl, the Cochrane } \\
\text { Library Database, the } \\
\text { Web of Knowledge, } \\
\text { the Applied Social } \\
\text { Sciences Index and } \\
\text { Abstracts, the } \\
\text { International } \\
\text { Bibliography of the } \\
\text { Social Sciences, } \\
\text { Sociological } \\
\text { Abstracts, the } \\
\text { Campbell } \\
\text { Collaboration and } \\
\text { grey literature } \\
\text { databases. } \\
\text { Articles published } \\
\text { January 1966 to April } \\
\text { 2005. } \\
\text { Search terms: All } \\
\text { related to } \\
\text { homosexual, lesbian } \\
\text { and bisexual people } \\
\text { and all related to } \\
\text { mental disorders, } \\
\text { suicide, and } \\
\text { deliberate self-harm. } \\
\text { Inclusion: Papers on } \\
\text { population based } \\
\text { studies which } \\
\text { contained concurrent } \\
\text { heterosexual } \\
\text { comparison groups } \\
\text { and valid definition of } \\
\text { sexual orientation } \\
\text { and mental health } \\
\text { outcomes. } \\
\text { Of } 13,706 \text { papers } \\
\text { identified, } 476 \text { were } \\
\text { initially selected and } \\
\text { Ondudies) met stiteria. } \\
\text { one stu met }\end{array}$ & $\begin{array}{l}\text { Systematic } \\
\text { review and } \\
\text { meta-analysis }\end{array}$ & $\begin{array}{l}\text { LGB people are at } \\
\text { higher risk of } \\
\text { suicidal behavior, } \\
\text { mental disorder and } \\
\text { substance misuse } \\
\text { and dependence } \\
\text { than heterosexuals. } \\
\text { The results of the } \\
\text { meta-analyses } \\
\text { demonstrate a } \\
\text { twofold excess in } \\
\text { risk of suicide } \\
\text { attempts in the } \\
\text { preceding year in } \\
\text { men and women, } \\
\text { and a fourfold } \\
\text { excess in risk in gay } \\
\text { and bisexual men } \\
\text { over a lifetime. } \\
\text { Similarly, } \\
\text { depression, anxiety, } \\
\text { alcohol and } \\
\text { substance misuse } \\
\text { were at least } 1.5 \\
\text { times more common } \\
\text { in LGB people. } \\
\text { Findings were } \\
\text { similar in men and } \\
\text { women but LB } \\
\text { women were at } \\
\text { particular risk of } \\
\text { substance } \\
\text { dependence, while } \\
\text { lifetime risk of } \\
\text { suicide attempts } \\
\text { was especially high } \\
\text { in GB men. }\end{array}$ \\
\hline
\end{tabular}

(C) The International Journal of Indian Psychology, ISSN 2348-5396 (e)| ISSN: 2349-3429 (p) | 108 
A Narrative Review of the Relationship between Victimization, Depression and Suicide Ideation among Lesbian, Gay and Bisexual Individuals

\begin{tabular}{|c|c|c|c|c|}
\hline Reference & Sample & Test & Design & Results \\
\hline & & $\begin{array}{l}\text { all our four quality } \\
\text { criteria and seven met } \\
\text { three of these criteria. }\end{array}$ & & \\
\hline Shields et al., 2012 & $\begin{array}{l}\text { United States } \\
\text { of America; } \\
2,154 \text { students } \\
\text { in grades } 9- \\
12 \text { across } 15 \\
\text { SFUSD high } \\
\text { schools. }\end{array}$ & $\begin{array}{l}\text { Three suicide risk- } \\
\text { related outcomes } \\
\text { from the Youth Risk } \\
\text { Behavior Survey } \\
\text { (YRBS) were } \\
\text { analyzed as } \\
\text { dichotomous } \\
\text { dependent variables: } \\
\text { (1) } \\
\text { Sadness/Depression, } \\
\text { (2) Suicide plan, (3) } \\
\text { Suicide attempt. } \\
\text { A five-item scale } \\
\text { measuring alcohol } \\
\text { and marijuana use: } \\
\text { Current alcohol use, } \\
\text { binge drinking, } \\
\text { drinking at school, } \\
\text { marijuana use, } \\
\text { marijuana use at } \\
\text { school. } \\
\text { A five-item scale } \\
\text { measuring } \\
\text { other drug use: } \\
\text { Lifetime use of } \\
\text { cocaine, inhalants, } \\
\text { heroin, } \\
\text { methamphetamines, } \\
\text { ecstasy. } \\
\text { A five-item scale } \\
\text { measuring } \\
\text { victimization: In past } \\
\text { 12 months - bullied at } \\
\text { school, skipped } \\
\text { school for safety } \\
\text { concerns, got in a } \\
\text { physical fight, injured } \\
\text { in a fight, threatened } \\
\text { or injured with a } \\
\text { weapon at school. }\end{array}$ & Survey & $\begin{array}{l}\text { Lesbian, gay, or } \\
\text { bisexual (LGB) } \\
\text { youth reported } \\
\text { significantly higher } \\
\text { rates of substance } \\
\text { use, victimization, } \\
\text { and suicide risk- } \\
\text { related outcomes } \\
\text { than heterosexual } \\
\text { youths. } \\
\text { However, in the } \\
\text { controlled } \\
\text { regression models, } \\
\text { victimization was a } \\
\text { significant predictor } \\
\text { of } \\
\text { sadness/depression } \\
\text { and suicide } \\
\text { attempts, regardless } \\
\text { of sexual } \\
\text { orientation. } \\
\text { There was a } \\
\text { significant } \\
\text { interaction effect } \\
\text { between sexual } \\
\text { orientation and } \\
\text { victimization on } \\
\text { suicide planning, } \\
\text { with heterosexual } \\
\text { youths more } \\
\text { affected than LGB } \\
\text { youths. }\end{array}$ \\
\hline
\end{tabular}

(c) The International Journal of Indian Psychology, ISSN 2348-5396 (e)| ISSN: 2349-3429 (p) | 109 


\section{A Narrative Review of the Relationship between Victimization, Depression and Suicide Ideation among Lesbian, Gay and Bisexual Individuals}

\begin{tabular}{|c|c|c|c|c|}
\hline Reference & Sample & Test & Design & Results \\
\hline & & $\begin{array}{l}\text { An item measuring } \\
\text { sexual orientation: } \\
\text { "Which of the } \\
\text { following best } \\
\text { describes you?" } \\
\text { (heterosexual; } \\
\text { gay/lesbian; bisexual; } \\
\text { not sure). }\end{array}$ & & \\
\hline Marshal et al., 2011 & $\begin{array}{l}\text { United States } \\
\text { of America; } \\
\text { mean age = } 18 \\
\text { years, and an } \\
\text { upper bound } \\
\text { of the age } \\
\text { range not } \\
\text { exceeding } 21 \\
\text { years. }\end{array}$ & $\begin{array}{l}\text { Inclusion: (1) } \\
\text { Reported rates of } \\
\text { depression and/or } \\
\text { suicidality among } \\
\text { sexual minority and } \\
\text { heterosexual youth, } \\
\text { (2) A sample mean } \\
\text { age of } \leq 18 \text { years, and } \\
\text { an upper bound of the } \\
\text { age range not } \\
\text { exceeding } 21 \text { years. } \\
\text { Databases: PsycInfo } \\
\text { and MedLine } \\
\text { Search terms: } \\
\text { Suicide, depression, } \\
\text { gay, lesbian, LGB, } \\
\text { adolescent. } \\
\text { A total of } 20 \text { suicide } \\
\text { and } 12 \text { depression } \\
\text { studies were } \\
\text { identified, resulting in } \\
24 \text { total studies with } \\
\text { seven of them } \\
\text { examining both } \\
\text { outcomes. }\end{array}$ & Meta-analysis & $\begin{array}{l}\text { SMY reported } \\
\text { significantly higher } \\
\text { rates of suicidality } \\
\text { (odds ratio [OR] = } \\
\text { 2.92) and } \\
\text { depression } \\
\text { symptoms } \\
\text { (standardized mean } \\
\text { difference, d = .33) } \\
\text { as compared with } \\
\text { the heterosexual } \\
\text { youth. } \\
\text { Disparities } \\
\text { increased with the } \\
\text { increase in the } \\
\text { severity of } \\
\text { suicidality (ideation } \\
\text { [OR = 1.96], } \\
\text { intent/plans [OR = } \\
\text { 2.20], suicide } \\
\text { attempts [OR = } \\
\text { 3.18], suicide } \\
\text { attempts requiring } \\
\text { medical attention } \\
\text { [OR = 4.17]). } \\
\text { Effects did not vary } \\
\text { across gender, } \\
\text { recruitment source, } \\
\text { and sexual } \\
\text { orientation } \\
\text { definition. }\end{array}$ \\
\hline
\end{tabular}




\section{A Narrative Review of the Relationship between Victimization, Depression and Suicide Ideation among Lesbian, Gay and Bisexual Individuals}

\section{DISCUSSION}

This narrative review identified sixteen studies in examining the relationship between victimization, depression, and suicide ideation among lesbian, gay, and bisexual (LGB) individuals.

\section{Victimization among sexual minorities}

Researchers agree that victimization can cause a highly stressful experience for its victim (O’Brennan et al., 2009; Rigby, 2003). Several studies have suggested that individuals perceived as sexual minorities (i.e., gay, lesbian, bisexual, and transgendered) are specific targets of victimization (Berlan et al., 2010; Williams et al., 2003). Patrick et al. (2013), in investigating the association between perceived sexual orientation, bullying, and quality of life among US adolescents, indicated that there is a substantial and significant impact of bullying on youth quality of life. Compared to their heterosexual counterparts, sexual minority individuals are more likely to report sexual minority-specific victimization (Burton et al., 2013; LeVasseur et al., 2013; Shields et al., 2012). For example, a study conducted among 351 racial/ethnic minority young men with a mean age of 20.4 years, who have sex with men, revealed that overall, $74.1 \%$ reported being made fun of because of their sexuality, 58\% reported being treated rudely or unfairly because of their sexuality, and $16.2 \%$ reported being hit or assaulted because of their sexuality, of which $4 \%$ experienced physical violence many times (Hightow-Weidman et al., 2011). It is important to note that there is a gender difference when it comes to the victimization of sexual minorities - males are more likely than females to report victimization because of both perceived sexual orientation and identified sexual orientation (Patrick et al., 2013; Russell et al., 2011). Furthermore, Mueller et al. (2015), in examining the intersections of sexual orientation, gender, and race/ethnicity, proposed that there is a racial difference in bullying among LGB youths - where white LGB youths were more likely to be bullied than their white same gender, heterosexual peers; however, black LGB youths, on the other hand, were no more vulnerable to bullying than their same gender white heterosexual peers.

\section{Victimization and depression among sexual minorities}

Sexual minorities are more vulnerable to poor mental health outcomes than sexual majorities (Mueller et al., 2015; King et al., 2008; Marshal et al., 2011, Russell et al., 2001; Patrick et al., 2013; Russell et al., 2011; Hightow-Weidman et al., 2011). Marshal et al. (2011), in studying the disparities between rates of depression symptoms in sexual minority and heterosexual youths, suggested that sexual minority youths experience significantly higher levels of depression symptoms than heterosexual youths. According to King et al. (2008), in understanding the association between LGB individuals and the risk of mental disorder, depression is at least 1.5 times more common in LGB individuals than in heterosexuals. These poorer mental health outcomes among sexual minorities, compared to sexual majorities, are associated with LGBT victimization (Russell et al., 2011; Burton et al., 2013). For example, High tow-Weidman et al. (2011), in assessing the relationship between sexual identity-based maltreatment and emotional 


\section{A Narrative Review of the Relationship between Victimization, Depression and Suicide Ideation among Lesbian, Gay and Bisexual Individuals}

distress among sexual minority young men, indicated that there is a significant association between experiencing a high level of sexually-related victimization and depressive symptomatology. Russell et al. (2011), in investigating the relationship between reports of LGBT school victimization and young adult psychosocial health, also revealed that LGBT young adults who experience victimization are 2.6 times more likely to report depression above the clinical cut-off (CES-D $\geq 16)$.

\section{Victimization and suicide ideation among sexual minorities}

Sexual minorities are more likely to report having suicide ideation and attempt than their heterosexual counterparts - this is associated with LGBT victimization (Russell et al., 2001; Blosnich \& Bossarte, 2012; LeVasseur et al., 2013; Russell et al., 2011; Hightow-Weidman et al., 2011; Burton et al., 2013; Muehlenkamp et al., 2015; Walls et al., 2010; Mueller et al., 2015; Ferlatte et al., 2015; Bergen et al., 2013; King et al., 2008; Shields et al., 2012). Russell et al. (2011), in investigating the relationship between reports of LGBT school victimization and young adult risk behavior, reported that LGBT young adults who experienced victimization were 5.6 times more likely to report having attempted suicide that required medical attention at least once. Ferlatte et al. (2015), in understanding the factors contributing to higher rates of suicide related ideation and behavior in Canadian gay and bisexual men, stated that $49.9 \%$ of the sample reported lifetime experiences of suicide ideation and attempt - this was over six times greater than that reported by Canadian heterosexual men. Even though suicide ideation among sexual minorities is associated with LGBT victimization, some studies propose that sexual minorities are still more likely to report having suicide ideation and attempt compared to heterosexual individuals even with victimization as well as other important explanatory variables (e.g., social stigma or a lack of social support) held constant (Mueller et al., 2015; Marshal et al., 2011). Furthermore, it is interesting to note that sexual minorities who are bisexual are more likely to report suicide ideation and attempt than sexual minorities who are not bisexual (Marshal et al., 2011; Blosnich and Bossarte, 2012). A double closet framework has been used in understanding elevated risk profiles among bisexuals; in that they must keep secret their homosexual activities, attractions or relationships from their heterosexual social groups, and conversely with their homosexual social groups (Blosnich and Bossarte, 2012).

\section{The role of depression in understanding suicide ideation among sexual minorities}

Studies suggest that symptoms of depression are correlated with a lifetime history of suicide ideation and attempt among LGBT individuals (Mustanski and Liu, 2012; Russell et al., 2001; Ferlatte et al., 2015). Russell et al. (2001), in examining adolescent sexual orientation and suicide risk, proposed that youths with same-sex sexual orientation, reporting elevated levels of suicidality, are more likely to feel depressed and hopeless. Ferlatte et al. (2015), in understanding the factors contributing to higher rates of suicide related ideation and behavior among Canadian gay and bisexual men, reported that when all health problems (i.e., smoking, party drugs, depression, anxiety, STIs, HIV risk and HIV) were included in a single model (multi-indicator), 


\section{A Narrative Review of the Relationship between Victimization, Depression and Suicide Ideation among Lesbian, Gay and Bisexual Individuals}

the association with suicide ideation and attempt was removed; but remained strongest for depression. This is not surprising since mood disorders are known to convey the highest risk for suicide related ideation and behavior in general populations (Ferlatte et al., 2015).

\section{LIMITATIONS}

There are several limitations in this narrative review. First, most of the studies conducted are cross-sectional and do not allow one to determine causal relationships. Second, because all of the studies are self-reported, it is hard to know to what extents sexual minorities over-report or under-report experiences of victimization, mental health problems, suicide thoughts and suicide attempts. Nevertheless, recall bias of these experiences is unlikely because these events are considered highly salient to sexual minorities. Furthermore, some sexual minorities may choose not to report these experiences because of social stigma or a fear of retaliation. Third, most of the studies do not include sexual minorities who are high school dropouts, jobless, incarcerated, homeless, or runaways; and, because sexual minority individuals are more likely to be homeless or runaways (Cochran et al., 2002), these studies may lack generalizability. Fourth, the sample of these studies is largely from the United States; and thus, the results obtained may not be generalizable outside this country. Finally, the studies that have random or census samples constitute national datasets as these samples came first from a convenience sample of institutions that elected to administer the survey. For this reason, there may be selection bias; in which organizations or institutions opt to participate, and therefore, it may not be appropriate to generalize the findings to a nationwide or international population.

\section{CONCLUSION}

This narrative review examines current existing literature and studies in discussing the relationship between victimization, depression, and suicide ideation and attempt among lesbian, gay, and bisexual (LGB) individuals. There is a consensus among researchers with respect to the notion that victimization represents a highly stressful experience for its victims (O'Brennan et al., 2009; Rigby, 2003). Several studies have suggested that individuals perceived as sexual minorities (i.e., gay, lesbian, bisexual, and transgendered), are specific target of victimization (Berlan et al., 2010; Williams et al., 2003). Compared to their heterosexual counterparts, sexual minority individuals are more likely to report sexual minority-specific victimization (Burton et al., 2013; LeVasseur et al., 2013; Shields et al., 2012). Furthermore, sexual minorities are more vulnerable to poor mental health outcomes (e.g., the symptoms of depression) than sexual majorities; and this is related to LGBT victimization (Mueller et al., 2015; King et al., 2008; Marshal et al., 2011, Russell et al., 2001; Patrick et al., 2013; Russell et al., 2011; HightowWeidman et al., 2011). Furthermore, sexual minorities are more likely to report having suicide ideation and attempt than heterosexuals and this is associated with LGBT victimization (Russell et al., 2001; Blosnich and Bossarte, 2012; LeVasseur et al., 2013; Russell et al., 2011; HightowWeidman et al., 2011; Burton et al., 2013; Muehlenkamp et al., 2015; Walls et al., 2010; Mueller et al., 2015; Ferlatte et al., 2015; Bergen et al., 2013; King et al., 2008; Shields et al., 2012).

(c) The International Journal of Indian Psychology, ISSN 2348-5396 (e)| ISSN: 2349-3429 (p) | 113 


\section{A Narrative Review of the Relationship between Victimization, Depression and Suicide Ideation among Lesbian, Gay and Bisexual Individuals}

Finally, several studies suggest that there is a significant correlation between symptoms of depression and a lifetime history of suicide ideation and attempt among LGBT individuals (Mustanski and Liu, 2012; Russell et al., 2001; Ferlatte et al., 2015).

Studying the prevalence of the relationship between victimization, depression, and suicide ideation and attempt among sexual minorities presents several implications. For example, assessing mental health and risk behavior of LGBT individuals, in relation to whether they have experienced victimization because of their sexual orientation, might help to identify specific areas to address in interventions. Furthermore, knowing the prevalence of victimization caused by one's sexual orientation, as well as the extent of depressive symptoms and suicide ideation present in a sexual minority community, is useful for policy makers and organizations interested in monitoring and evaluating prevention efforts.

It is easy to pathologize the entire population when studying health (including mental health) disparities in an at-risk population such as sexual minorities. Nevertheless, it is important to point out that in the larger population, there are sexual minority individuals who are welladjusted and have no significant symptoms of depression or history of suicidality. This suggests that resilience can be found in every at-risk population. Some studies have identified protective factors such as Gay Straight Alliances in schools (Hatzenbuehler, 2011) and family acceptance (Ryan et al. 2010). Future research should explore resilience factors that are proven to protect sexual minority individuals in order to develop effective programs and interventions in improving the mental health of these sexual minorities. Last but not least, similar studies are encouraged to be conducted or replicated in other countries with local population sample for comparative purposes.

\section{Acknowledgments}

The author appreciates all those who participated in the study and helped to facilitate the research process.

\section{Conflict of Interests}

The author declared no conflict of interests.

\section{REFERENCES}

Berlan, E., Corliss, H., Field, A., Goodman, E., \& Austin, S. (2010). Sexual orientation and bullying among adolescents in the growing up today study. Journal of Adolescent Health, 46(4), 366-371.

Bontempo, D., \& D'augelli, A. (2002). Effects of at-school victimization and sexual orientation on lesbian, gay, or bisexual youths' health risk behavior. Journal of Adolescent Health, 30(5), 364-374. 


\section{A Narrative Review of the Relationship between Victimization, Depression and Suicide Ideation among Lesbian, Gay and Bisexual Individuals}

Brunstein Klomek, A., Sourander, A., \& Gould, M. (2010). The association of suicide and bullying in childhood to young adulthood: A review of cross-sectional and longitudinal research findings. Canadian Journal of Psychiatry, 55(2), 282-288.

Cochran, B. N., Stewart, A. J., Ginzler, J. A., Cauce, A. M. (2002). Challenges faced by homeless sexual minorities: Comparison of gay, lesbian, bisexual, and transgender homeless adolescents with their heterosexual counterparts. American Journal of Public Health. 2002, 92(5), 773-777.

D'augelli, A., Pilkington, N., \& Hershberger, S. (2002). Incidence and mental health impact of sexual orientation victimization of lesbian, gay, and bisexual youths in high school. School Psychology Quarterly, 17(2), 148-167.

Eisenberg, M., \& Aalsma, M. (2005). Bullying and peer victimization: Position paper of the society for adolescent medicine. Journal of Adolescent Health, 36, 88-91.

Espelage, D. L., Aragon, S. R., Birkett, M., \& Koening, B. W. (2008). Homophobic teasing, psychological outcomes, and sexual orientation among high school students: What influence do parents and schools have? School Psychology Review, 37(2), 202-216.

Harris Interactive, Gay, Lesbian \& Straight Education Network. (2005). From teasing to torment: school climate in America, a survey of students and teachers. New York, NY: Gay, Lesbian \& Straight Education Network.

Hatzenbuehler, M. L. (2011). The social environment and suicide attempts in lesbian, gay, and bisexual youth. Pediatrics, 127, 896-903.

Meyer, I. (1995). Minority stress and mental health in gay men. Journal of Health and Social Behavior, 36, 38-38.

O'brennan, L., Bradshaw, C., \& Sawyer, A. (2009). Examining developmental differences in the social-emotional problems among frequent bullies, victims, and bully/victims. Psychology in the Schools, 46(2), 100-115.

Rigby, K. (2003). Consequences of bullying in schools. Canadian Journal of Psychiatry, 48(9), 583-590.

Ryan, C., Russell, S. T., Huebner, D., Diaz, R., \& Sanchez, J. (2010). Family acceptance in adolescence and the health of LGBT adults. Journal of Child and Adolescent Psychiatric Nursing, 23, 205-213.

Shields, J., Whitaker, K., Glassman, J., Franks, H.,\& Howard, K. (2012). Impact of victimization on risk of suicide among lesbian, gay, and bisexual high school students in San Francisco. Journal of Adolescent Health, 50(4), 418-420.

Wilkinson, L., \& Pearson, J. (2009). School culture and the well-being of same-sex-attracted youth. Gender \& Society, 23(4), 542-568.

Williams, T., Connolly, J., Pepler, D., \& Craig, W. (2003). Questioning and sexual minority adolescents: High school experiences of bullying, sexual harassment and physical abuse. Canadian Journal of Community Mental Health, 22(2), 47-58. 


\section{A Narrative Review of the Relationship between Victimization, Depression and Suicide Ideation among Lesbian, Gay and Bisexual Individuals}

\section{Studies included in the narrative review}

Blosnich, J., \& Bossarte, R. (2012). Drivers of disparity: Differences in socially based risk factors of self-injurious and suicidal behaviors among sexual minority college students. Journal of American College Health, 60(2), 141-149.

Burton, C. M., Marshal, M. P., Chisolm, D. J., Sucato, G. S., \& Friedman, M. S. (2013). Sexual minority-related victimization as a mediator of mental health disparities in sexual minority youth: A longitudinal analysis. Journal of Youth and Adolescence, 42(3), 394402.

Ferlatte, O., Dulai, J., Hottes, T. S., Trussler, T., \& Marchand, R. (2015). Suicide related ideation and behavior among Canadian gay and bisexual men: A syndemic analysis. BMC Public Health, 15(1), 1-9.

Hightow-Weidman, L. B., Phillips, G., Jones, K. C., Outlaw, A. Y., Fields, S. D., \& Smith, F. C. (2011). Racial and sexual identity-related maltreatment among minority YMSM: Prevalence, perceptions, and the association with emotional distress. AIDS Patient Care \& Stds, 25, S39-S45.

King, M., Semlyen, J., Tai, S., Killaspy, H., Osborn, D., Popelyuk, D., \& Nazareth, I. (2008). A systematic review of mental disorder, suicide, and deliberate self-harm in lesbian, gay and bisexual people. BMC Psychiatry, 8, 70.

Levasseur, M. T., Kelvin, E. A., \& Grosskopf, N. A. (2013). Intersecting identities and the association between bullying and suicide attempt among New York City youths: Results from the 2009 New York City youth risk behavior survey. American Journal of Public Health, 103(6), 1082-1089.

Marshal, M., Dietz, L., Friedman, M., Stall, R., Smith, H., Mcginley, J., Thoma, C., Murray, J., D’augelli, R., Brent, D. (2011). Suicidality and depression disparities between sexual minority and heterosexual youth: A meta-analytic review. Journal of Adolescent Health, 49, 115-123.

Mueller, A. S., James, W., Abrutyn, S., \& Levin, M. L. (2015). Suicide ideation and bullying among US adolescents: Examining the intersections of sexual orientation, gender, and race/ethnicity. American Journal of Public Health, 105(5), 980-985.

Muehlenkamp, J., Hilt, L., Ehlinger, P., \& Mcmillan, T. (2015). Nonsuicidal self-injury in sexual minority college students: A test of theoretical integration. Child and Adolescent Psychiatry and Mental Health, 9, 16.

Mustanski, B., \& Liu, R. (2013). A longitudinal study of predictors of suicide attempts among lesbian, gay, bisexual, and transgender youth. Archives of Sexual Behavior, 42(3), 437448.

Patrick, D. L., Bell, J. F., Huang, J. Y., Lazarakis, N. C., \& Edwards, T. C. (2013). Bullying and quality of life in youths perceived as gay, lesbian, or bisexual in Washington State, 2010. American Journal of Public Health, 103(7), 1255-1261. 


\section{A Narrative Review of the Relationship between Victimization, Depression and Suicide Ideation among Lesbian, Gay and Bisexual Individuals}

Russell, S. T., \& Joyner, K. (2001). Adolescent sexual orientation and suicide risk: Evidence from a natural study. American Journal of Public Health, 91(8), 1276-1281.

Russell, S. T., Ryan, C., Toomey, R. B., Diaz, R. M., \& Sanchez, J. (2011). Lesbian, gay, bisexual, and transgender adolescent school victimization: Implications for young adult health and adjustment. Journal of School Health, 81(5), 223-230.

Shields, J., Whitaker, K., Glassman, J., Franks, H.,\& Howard, K. (2012). Impact of victimization on risk of suicide among lesbian, gay, and bisexual high school students in San Francisco. Journal of Adolescent Health, 50, 418-420.

Van Bergen, D. D., Bos, H. W., van Lisdonk, J., Keuzenkamp, S., \& Sandfort, T. M. (2013). Victimization and suicidality among Dutch lesbian, gay, and bisexual youths. American Journal of Public Health, 103(1), 70-72.

Walls, N. E., Laser, J., Nickels, S. J., \& Wisneski, H. (2010). Correlates of cutting behavior among sexual minority youths and young adults. Social Work Research, 34(4), 213-226.

How to cite this article: M Aen, Ibrahim N, Din N (2017), A Narrative Review of the Relationship between Victimization, Depression and Suicide Ideation among Lesbian, Gay and Bisexual Individuals, International Journal of Indian Psychology, Volume 4, Issue 2, No. 94, ISSN:2348-5396 (e), ISSN:2349-3429 (p), DIP:18.01.150/20170402, ISBN:978-1-365-84229-0 